\title{
Magnetic Properties of Percolated Perpendicular FePt-MgO Films
}

\author{
An-Cheng Sun ${ }^{1}$, Jen-Hwa Hsu ${ }^{1}$, P. C. Kuo ${ }^{2}$, and H. L. Huang ${ }^{1}$ \\ ${ }^{1}$ Department of Physics and Center for Nanostorage Research, National Taiwan University, Taipei 106, Taiwan \\ ${ }^{2}$ Institute of Materials Science and Engineering, National Taiwan University, Taipei 106, Taiwan
}

\begin{abstract}
Percolated perpendicular FePt-MgO films with a $\left(\mathrm{Fe}_{48} \mathrm{Pt}_{52}\right)_{100-x}-(\mathrm{MgO})_{x} / \mathrm{Pt}(001) / \mathrm{Cr}(002)$ structure were prepared by conventional dc magnetron sputtering $(x=0-6.13)$. Magnetic measurements demonstrate that the coercivity of the magnetic film drastically increases from 169 to $285 \mathrm{kA} / \mathrm{m}$ as the MgO content is increased from 0 to $0.15 \mathrm{vol}$.\%. However, the grain sizes of the FePt phase do not significantly varying upon doping with $\mathrm{MgO}$. MgO does not appear at the grain boundaries of the FePt phase, but is present as crystalline dots that are uniformly precipitated in the FePt matrix. The MFM images revealed that the domain structure transformed from extending to isolate when the MgO dots precipitated into the FePt grains. Consequently, the MgO dots serve as pinning sites of the domain wall and enhance perpendicular coercivity. Percolated perpendicular magnetic recording is thus regarded as a solution to the problem of thermal instability in ultrasmall grains.
\end{abstract}

Index Terms-Coercive force, FePt alloy, percolated meida, perpendicular magnetic recording, precipitation.

\section{INTRODUCTION}

$\mathbf{T}$ HE granular perpendicular medium (GPM) can provide improved recording density with higher thermal stability and better recording resolution [1]-[3]. However, in GPM, the issue of medium noise still critically limits the recording density when the dominant source of medium noise is transition jitter noise [2], [3]. The magnetic grain size must be further scaled down into the nanoscaled range to reduce transition jitter noise while simultaneously maintaining a sufficient medium signal-to-noise ratio (SNR) in an ultra-high density magnetic recording medium [2]-[5]. However, as the magnetic grain size decreases, the thermal magnetic stability is normally degraded. Therefore, the idea of the percolated perpendicular medium (PPM) was recently proposed to solve this issue [6]. The PPM comprises closely packed grains with relatively strong intergranular exchange coupling and well-separated nanometer-sized nonmagnetic columns, or dots, referred to as pinning sites. In this study, perpendicular magnetic FePt media, consisting of well-separated insulating nonmagnetic $\mathrm{MgO}$ nanoparticles, were prepared to test this idea. Incorporating $\mathrm{MgO}$ nanoparticles into the FePt matrix increases its coercivity. The magnetic hardness mechanism involves switching from domain wall motion in a $\operatorname{FePt}(001)$ film to domain wall pinning in percolated $\mathrm{FePt}-\mathrm{MgO}$ films. Similar investigations have been reported by Suzuki et al. [7], [8]. However, in their study, $\mathrm{MgO}$ of 30 vol.\% was doped in the FePt phase and the network structure consisting of rectangular $\mathrm{MgO}$ and faceted $\mathrm{FePt}$ grains was formed. This type of structure is not exactly identical to PPM as proposed previously. Moreover, in our investigation, when a small amount of $\mathrm{MgO}$ is incorporated into FePt film, $\mathrm{MgO}$ dots behave similar to the nanoinclusions and precipitated in the inner FePt grain. Consequently, the PPM microstructure with randomly distributed nonmagnetic $\mathrm{MgO}$ pinning sites in

Digital Object Identifier 10.1109/TMAG.2007.892999

Color versions of one or more of the figures in this paper are available online at http://ieeexplore.ieee.org. our investigation thus effectively enables reduction of transition noise without downgrading the thermal stability.

\section{EXPERIMENT}

Percolated $\left(\mathrm{Fe}_{48} \quad \mathrm{Pt}_{52}\right)_{100-\mathrm{x}}-(\mathrm{MgO})_{\mathrm{x}}$ film (where $\mathrm{x}=0-6.13 \mathrm{vol} . \%)$ was deposited on a textured $\mathrm{Pt}(001) /$ $\mathrm{Cr}(002)$ bilayer film with 7059 glass substrate in an ultra-high vacuum sputtering chamber. Before the percolated FePt-MgO films were deposited, the substrate was heated to $350{ }^{\circ} \mathrm{C}$ to prepare the $\mathrm{Cr}(002)$ underlayer and the $\operatorname{Pt}(001)$ buffer layer, and then to $420{ }^{\circ} \mathrm{C}$ to deposit $\mathrm{FePt}-\mathrm{MgO}$ films. The thicknesses of the $\mathrm{FePt}-\mathrm{MgO}$ film, the $\mathrm{Pt}$ buffer layer, and the $\mathrm{Cr}$ underlayer were set to 20,3, and $70 \mathrm{~nm}$, respectively. Various rf sputtering powers of $\mathrm{MgO}$ were used to vary the $\mathrm{MgO}$ content. The crystalline phases and microstructures of the films were identified by X-ray diffraction (XRD) using $\mathrm{Cu}-\mathrm{K}_{\alpha}$ radiation and high-resolution transmission electron microscopy (HRTEM), respectively. The chemical composition of the magnetic FePt layer was determined by energy dispersive spectroscopy (EDS), and the composition of the FePt was $\mathrm{Fe}_{48} \mathrm{Pt}_{52}$. Since $\mathrm{MgO}$ does not alloy with the magnetic FePt phase, the $\mathrm{MgO}$ content of the magnetic FePt matrix was determined by calculating $\left[R_{\mathrm{MgO}} /\left(R_{\mathrm{FePt}}+R_{\mathrm{MgO}}\right)\right] \times 100 \mathrm{vol} . \%$, where $R$ denotes the sputtering rate. The thicknesses of the film were measured by atomic force microscopy (AFM). Magnetic properties were measured using a vibrating sample magnetometer (VSM) at room temperature with a maximum applied field of $\sim 1000$ $\mathrm{kA} / \mathrm{m}$. Domain structures were observed using a magnetic force microscopy (MFM) in an ac-demagnetized state.

\section{RESULTS AND DisCUSSION}

Fig. 1(a) displays the X-ray diffraction patterns of $\left(\mathrm{Fe}_{48}\right.$ $\left.\mathrm{Pt}_{52}\right)_{100-x}-(\mathrm{MgO})_{x} / \mathrm{Pt} / \mathrm{Cr}$ trilayer films with various $\mathrm{MgO}$ contents. Except for the peak of the $\mathrm{Cr}(002)$ underlayer, all visible peaks are attributed to the fcc or the $L 1_{0} \mathrm{FePt}$ phase, indicating that no new phase was formed. Since the $\mathrm{MgO}$ content is low, the peaks that correspond to $\mathrm{MgO}$ were not observed. Additionally, an excellent ordered $\mathrm{FePt}(001)$ texture without $\mathrm{FePt}(111)$ and (200) peaks is present when $\mathrm{MgO}$ is absent, revealing that a pure $\mathrm{FePt}(001)$ film with good perpendicular magnetic anisotropy property was obtained [9]. In Fig. 1(a), the intensity 


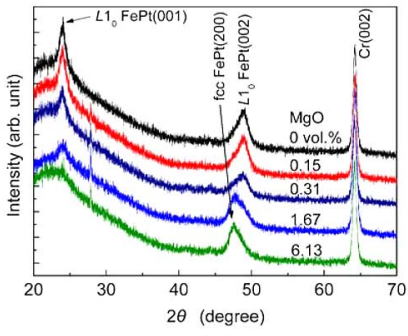

(a)

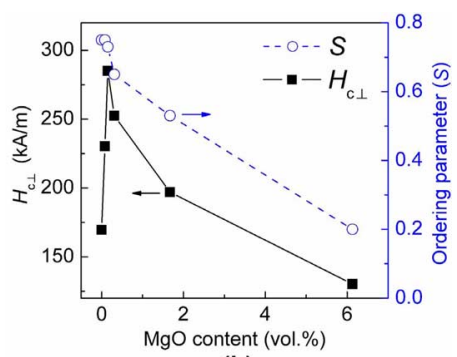

(b)
Fig. 1. (a) X-ray diffraction patterns and (b) out-of-plane coercivity $\left(H_{\mathrm{c}} \perp\right)$ and long-range ordering parameter $(S)$ of $\left(\mathrm{Fe}_{48} \mathrm{Pt}_{52}\right)_{100-\mathrm{x}}-(\mathrm{MgO})_{\mathrm{x}} / \mathrm{Pt} / \mathrm{Cr}$ trilayer films with various amounts of $\mathrm{MgO}$ contents.

of the $L 1_{0}$ FePt-ordered peaks was predominant, and then fell as the $\mathrm{MgO}$ content increased. The ordered $L 1_{0} \mathrm{FePt}(002)$ peak transformed into the disordered fcc FePt(200) peak and the ordered $\mathrm{FePt}(001)$ peak disappeared when $\mathrm{MgO}$ reached 6.13 vol.\%. The appearance of the disordered FePt(200) peak suggests a degraded (001) texture of the FePt phase. Fig. 1(b) plots the out-of-plane coercivity $\left(H_{\mathrm{c}} \perp\right)$ and long-range ordering parameter $(S)$ versus $\mathrm{MgO}$ content. $H_{\mathrm{c}} \perp$ first increased markedly as the $\mathrm{MgO}$ content increased from 0 to $0.15 \mathrm{vol} . \%$, and then declined significantly as the amount of $\mathrm{MgO}$ increased. A peak value of $H_{\mathrm{c}} \perp \sim 285 \mathrm{kA} / \mathrm{m}$ was found at $\mathrm{MgO}=0.15 \mathrm{vol} . \%$. However, $S$ remained the same, at approximately 0.74 , at a $\mathrm{MgO}$ content of between 0 and $0.15 \mathrm{vol} \%$; it then decreases rapidly as the $\mathrm{MgO}$ content increases. Therefore, the drop in $H_{\mathrm{c}} \perp$ when $\mathrm{MgO}>0.31$ vol.\% is primarily associated with the decline in the ordering parameter $S$. However, the dependences of $H_{\mathrm{c}} \perp$ and $S$ on $\mathrm{MgO}$ content between 0 and 0.15 vol.\% are not similar. This finding is rather unexpected. The enhancement in hysteresis coercivity is typically caused by an improvement in ordering [10], [11]. Thus, the large increase of $H_{c} \perp$ with the incorporation of a small amount of $\mathrm{MgO}$ should have another origin, which will be discussed later.

Comparing the integrated intensities and positions of $\mathrm{FePt}(001)$ and (002) peaks from FePt film with those from the composite $(\mathrm{FePt})_{99.85}-(\mathrm{MgO})_{0.15}$ film, as displayed in Fig. 1(a), reveals no detectable difference between the film structures, suggesting that both had similar alignments. However, a large difference between $H_{\mathrm{c}} \perp$ values was discovered, implying that the microstructure and magnetization behavior of both films may differ [12]. The microstructure of the composite $(\mathrm{FePt})_{99.85}-(\mathrm{MgO})_{0.15}$ film was examined to elucidate the role of $\mathrm{MgO}$ in the FePt matrix. For comparison, the microstructure of FePt film was also studied. Fig. 2(a) and (b) presents plane-view TEM bright field images. The images revealed that doping with $\mathrm{MgO}$ does not significantly change the grain sizes of the FePt phase. The plane-view TEM bright field images reveal that many white $\mathrm{MgO}$ dots (identified with TEM-EDS) were well separated throughout the FePt matrix in $(\mathrm{FePt})_{99.85}-(\mathrm{MgO})_{0.15} / \mathrm{Pt} / \mathrm{Cr}$ trilayer films. The inset in Fig. 2(a) presents the lattice structure of $\mathrm{MgO}$ dots of the dotted circle in Fig. 2(a). Undoubtedly, the MgO dots are crystalline with a diameter of about $3 \mathrm{~nm}$. A better lattice matching at the $\mathrm{FePt} / \mathrm{MgO}$ interface was also observed; $\mathrm{MgO}$ may be precipitated inside the FePt grains, unlike in GPM films, in
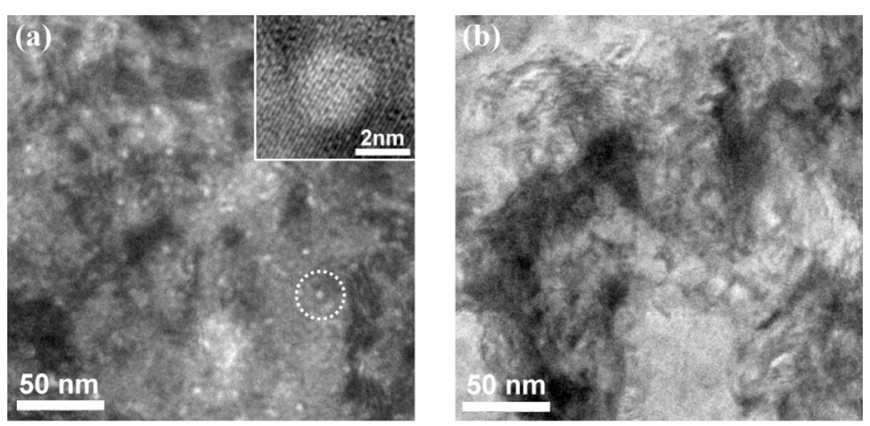

Fig. 2. Plane view TEM images of (a) $(\mathrm{FePt})_{99.85}-(\mathrm{MgO})_{0.15} / \mathrm{Pt} / \mathrm{Cr}$ and (b) $\mathrm{FePt} / \mathrm{Pt} / \mathrm{Cr}$ trilayer films, respectively. The inset in Fig. 3(a) is the lattice structure of the $\mathrm{MgO}$ dot located within the dotted circle of Fig. 3(a).

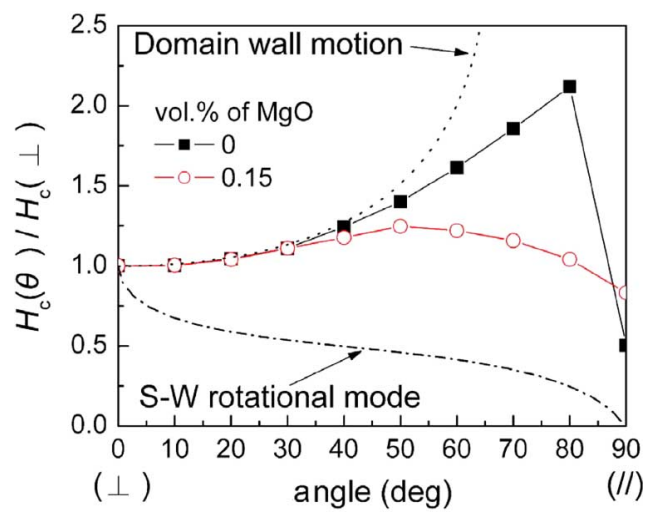

Fig. 3. Angular variation of the coercivity of $\mathrm{FePt} / \mathrm{Pt} / \mathrm{Cr}$ and $(\mathrm{FePt})_{99.85}-\mathrm{MgO}_{0.15} / \mathrm{Pt} / \mathrm{Cr}$ trilayer films.

which nonmagnetic insulator material, such as $\mathrm{Al}_{2} \mathrm{O}_{3}$ [13] or $\mathrm{SiO}_{2}$ [14], [15], is preferred to segregate at magnetic grain boundaries. However, in the case of the FePt-MgO film, $\mathrm{MgO}$ dots were surrounded by the FePt phase, as in the percolated film. As shown in Fig. 2(b), white dots were absent from the image of the $\mathrm{FePt} / \mathrm{Pt} / \mathrm{Cr}$ trilayer film.

The angular variation of the coercivity was determined to understand the magnetization reversal process. The results are plotted in Fig. 3. The coercivities at $0^{\circ}$ and $90^{\circ}$ are related to applied field normal and parallel to the film plane, respectively. Generally, for domain wall motion mechanism, the coercivity at $\theta$ should be proportional to $H / \cos (\theta)$, where $\theta$ is the angle between the applied field and the easy axis of the uniaxial anisotropy and $H$ is the coercivity when the applied field is in the direction of the easy axis. For comparison, the variation of coercivity with the angle $\theta$ for the rotation mechanism derived from the Stoner-Wohlfarth model was also illustrated in Fig. 3. As shown in Fig. 3, the magnetization reversal behavior of the $\mathrm{FePt} / \mathrm{Pt} / \mathrm{Cr}$ trilayer film is close to the domain wall motion over a wide-angle region, suggesting a strong coupling between magnetic domains [16]. The domain wall moves progressively through the magnetic grains. However, when the $\mathrm{MgO}$ dots were segregated into inner FePt grains, the domain wall motion was impeded by $\mathrm{MgO}$ dots and became discontinuously propagated. The $\mathrm{MgO}$ dots play a role of pinning sites to retard the domain wall motion. As a result, the magnetization reversal tends to be closer to the rotation mode as indicated in Fig. 3. 


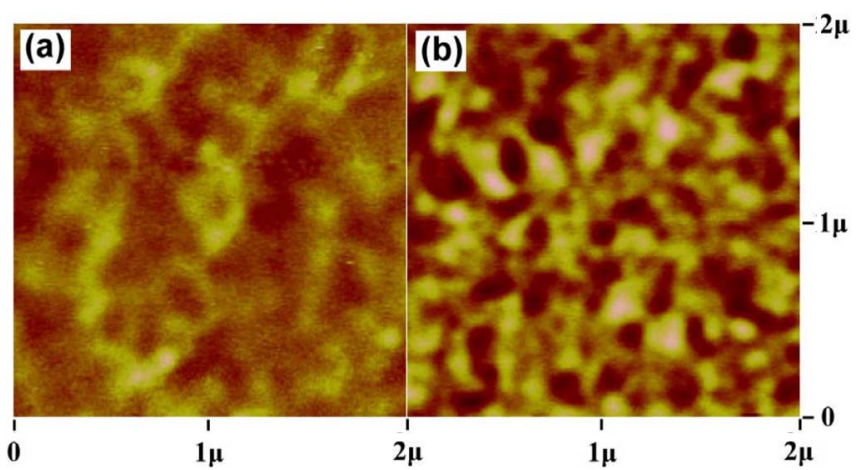

Fig. 4. Domain structures of (a) $\mathrm{FePt} / \mathrm{Pt} / \mathrm{Cr}$ and (b) $(\mathrm{FePt})_{99.85}-(\mathrm{MgO})_{0.15}$ $/ \mathrm{Pt} / \mathrm{Cr}$ trilayer films.

Fig. 4(a) and (b) illustrated the magnetic domain structures of FePt and $(\mathrm{FePt})_{99.85}-(\mathrm{MgO})_{0.15}$ films in an ac-demagnetized state, respectively. In Fig. 4(a), the extending domains were found. The average domain size was $250-300 \mathrm{~nm}$, and the boundaries were blurred. However, when $\mathrm{MgO}$ precipitated in the FePt grains, the domain size was reduced to $150-200 \mathrm{~nm}$ as shown in Fig. 4(b) and the boundaries were easily observable, revealing the existence of pinning sites introduced by $\mathrm{MgO}$ dots because the domain walls are located at the energy barrier ( $\mathrm{MgO}$ dots herein) during demagnetization [17]. The situation is the opposite of that in FePt film, in which the domain wall moves a long distance with few hindrances during the demagnetization process. Consequently, the extending domain was formed with a blurred boundary. This fact strongly indicates that the domain wall pinning effect occurs in $\mathrm{MgO}$-doped $\mathrm{FePt}$ thin film.

Since $\mathrm{MgO}$ dots are embedded in FePt matrix, these nano-size inclusions will act as major hindering sites to the domain wall motion and, consequently, enhances the coercivity $H_{\mathrm{c}} \perp$. On the other hand, microstress caused by the lattice mismatch between $\mathrm{FePt}(001)$ and $\mathrm{MgO}(002)$, which is roughly around 9.0 $\%$ [7], also existing around $\mathrm{MgO}$ dots, may cause an additional hindrance and further increase coercivity. However, further increasing the $\mathrm{MgO}$ content to more than 0.31 vol.\% converts PPM into GPM, since the excess $\mathrm{MgO}$ is segregated at $\mathrm{FePt}$ grain boundaries. Furthermore, increasing the $\mathrm{MgO}$ content will raise the ordering temperature, inhibiting the formation of ordered $L 1_{0} \operatorname{FePt}(001)$ phase [18], as shown in Fig. 1.

\section{CONCLUSION}

Percolated FePt-MgO films were prepared on a textured $\mathrm{Pt}(001) / \mathrm{Cr}(002)$ bilayer film using the 7059 glass substrate. $\mathrm{MgO}$ dots were uniformly precipitated in the FePt matrix and acted as pinning sites to the domain wall motion, causing an enhancement of $H_{\mathrm{c}} \perp$ when a small amount of $\mathrm{MgO}$ was doped into FePt grains. This fact demonstrates the benefits of using the percolated perpendicular medium to further increase the storage density of magnetic recording.

\section{ACKNOWLEDGMENT}

This work was supported by the Ministry of Economic Affairs of Taiwan under Contract 95-EC-17-A-08-S1-0006.

\section{REFERENCES}

[1] W. H. Jiang, H. Muraoka, Y. Sugita, and Y. Nakamura, "Thermal relaxation in perpendicular double-layered media," IEEE Trans. Magn., vol. 34, no. 4, pp. 1645-1647, Jul. 1998.

[2] A. Takeo, Y. Takahashi, Y. Tanaka, K. Miura, H. Muraoka, and Y. Nakamura, "Precise noise characterization of perpendicular recording media," J. Appl. Phys., vol. 87, pp. 4987-4989, May 2000.

[3] M. Hashimoto, K. Miura, H. Muraoka, H. Aoi, and Y. Nakamura, "Influence of magnetic cluster size distribution on SNR and bit error rate in perpendicular magnetic recording," J. Magn. Magn. Mater, vol. 287, pp. 123-127, Feb. 2005.

[4] S. Ishio, T. Wasiya, H. Saito, J. Bai, and W. Pei, "Magnetic reversal field map combined with medium noise analysis in $\mathrm{CoCrPt}-\mathrm{SiO}_{2}$ granular perpendicular recording medium," J. Appl. Phys., vol. 99, p. 093907, May 2006.

[5] S. J. Greaves, H. Muraoka, and Y. Kanai, "Discrete track media for 600 Gbits/in² recording," J. Appl. Phys., vol. 99, p. 08F903, Apr. 2006.

[6] J.-G. Zhu and Y. Tang, "A medium microstructure for high area density perpendicular recording," J. Appl. Phys., vol. 99, p. 08Q903, Apr. 2006.

[7] T. Suzuki and K. Ouchi, "Sputter-deposited (Fe-Pt)-MgO composite films for perpendicular recording media," IEEE Trans. Magn., vol. 37, no. 4, pp. 1283-1285, Jul. 2001.

[8] T. Suzuki, H. Muraoka, Y. Nakamura, and K. Ouchi, "Design and recording properties of $\mathrm{Fe}-\mathrm{Pt}$ perpendicular media," IEEE Trans. Magn., vol. 39, no. 2, pp. 691-696, Mar. 2003.

[9] A.-C. Sun, P. C. Kuo, J.-H. Hsu, H. L. Huang, and J.-M. Sun, "Epitaxial growth mechanism of $L 1_{0} \mathrm{FePt}$ thin films on $\mathrm{Pt} / \mathrm{Cr}$ bilayer with amorphous glass substrate," J. Appl. Phys., vol. 98, p. 076109, Oct. 2005.

[10] J. Wan, Y. Huang, Y. Zhang, M. J. Bonder, and G. C. Hadjipanayis, "Particulate FePt/Ag(C) films with strong perpendicular anisotropy," J. Appl. Phys., vol. 97, p. 10J121, May 2005.

[11] Y. F. Ding, J. S. Chen, E. Liu, and J. P. Wang, "Dependence of microstructure and magnetic properties of FePt films on $\mathrm{Cr}_{90} \mathrm{Ru}_{10}$ underlayers," J. Magn. Magn. Mater., vol. 285, pp. 443-449, Sep. 2005.

[12] J. D. Livingston, "A review of coercivity mechanisms," J. Appl. Phys., vol. 52, pp. 2544-2548, Mar. 1981.

[13] Y. K. Tahahashi and K. Hono, "Interfacial disorder in the $L 1_{0} \mathrm{FePt}$ particles capped with amorphous $\mathrm{Al}_{2} \mathrm{O}_{3}$," Appl. Phys. Lett., vol. 84, pp. 383-385, Jan. 2004.

[14] J. Z. Shi, S. N. Piramanayagam, C. S. Mah, H. B. Zhao, J. M. Zhao, Y. S. Kay, and C. K. Pock, "Influence of dual-Ru intermediate layers on magnetic properties and recording performance of $\mathrm{CoCrPt}-\mathrm{SiO}_{2}$ perpendicular recording media," Appl. Phys. Lett., vol. 87, p. 222503, Nov. 2005.

[15] G. Sáfrán, T. Suzuki, K. Ouchi, P. B. Barna, and G. Randnóczi, "Nanostructure formation of $\mathrm{Fe}-\mathrm{Pt}$ perpendicular magnetic recording media co-deposited with $\mathrm{MgO}, \mathrm{Al}_{2} \mathrm{O}_{3}$, and $\mathrm{SiO}_{2}$ additives," Thin Solid Films, vol. 496, pp. 580-584, Oct. 2006.

[16] T. Suzuki, N. Honda, and K. Ouchi, "Magnetization reversal process in polycrystalline ordered Fe-Pt(001) thin films," J. Appl. Phys., vol. 85, pp. 4301-4303, Apr. 1999.

[17] R. D. McMichael, F. Vajda, and E. Della-Torre, "Demagnetized-state dependence of henkel plots. II. Domain wall motion," J. Appl. Phys., vol. 75, pp. 5692-5694, May 1994.

[18] C. P. Luo and D. J. Sellmyer, "Structural and magnetic properties of $\mathrm{FePt}: \mathrm{SiO}_{2}$ granular thin films," Appl. Phys. Lett., vol. 75, pp. 3162-3164, Nov. 1999.

Manuscript received October 31, 2006; revised February 8, 2007 (e-mail: jhhsu@phys.ntu.edu.tw). 